\title{
Toward Rotavirus Vaccination Update
}

\author{
Masoud Mardani (ii) ${ }^{1}{ }^{*}$ \\ ${ }^{1}$ Infectious Diseases and Tropical Medicine Research Center, Shahid Beheshti University of Medical Sciences, Tehran, Iran \\ "Corresponding author: Infectious Diseases and Tropical Medicine Research Center, Shahid Beheshti University of Medical Sciences, Tehran, Iran. Tel: +98-9121132678, Email: \\ drmasoudmardani@yahoo.com
}

Received 2020 January 25; Accepted 2020 January 26.

Keywords: Rotavirus, Vaccination, Children

Rotavirus gastroenteritis is brought about by rotavirus that taints the stomach and the gut. This virus is normal in newborn children as well as young children, and kids under the age of five, particularly those between a half year and two years, are generally defenseless against the malady (1).

Rotavirus is exceptionally contagious among kids. Rehash diseases with various viral strains are conceivable, and most children have a few scenes of rotavirus contamination in the first long stretches of life. The primary contamination will, in general, be the most serious as the body develops insusceptibility protection from the infection thereafter (1).

The virus can spread quickly in a daycare center or household, primarily by the fecal-oral route, often when toddlers put their finger or a contaminated object in their mouths. The virus is stable in the environment and can live on contaminated surfaces or objects for up to 10 days, and occasionally lives in water or food. The virus is widespread in developing and developed countries (1).

Rotavirus immunizations were presented in five nations in the Eastern Mediterranean area as a major aspect of the national EPI plan. Monovalent rotavirus immunization is managed orally in a two-dose schedule while predominant rotavirus antibody is regulated orally in a threedose schedule plan.

The primary portion of the antibody should be directed during the time of about a month and a half to 15 weeks of age. Resulting dosages should be directed at four multi week interims. The most extreme age for directing the last portion of the antibody is prescribed to be at 32 weeks of age (1).

Rotavirus vaccines are safe and effective at preventing rotavirus disease. Both rotavirus vaccines are more than $85 \%$ effective at preventing severe rotavirus gastroenteritis. During the first year of the child's life, the rotavirus vaccine provides $85 \%$ - $98 \%$ protection against severe rotavirus disease, resulting in hospitalization and 74\% - 87\% protection against rotavirus disease of any severity. Rotavirus immunizations are protected, yet there is a little hazard for intussusception. The rotavirus antibodies cause an expected one extra instance of intussusception for each 20000 - 100000 US babies. Given the huge decrease in rotavirus malady, the advantages of rotavirus inoculation are considered to exceed the little expanded hazard for intussusception $(1,2)$.

Neither the antibody nor characteristic contamination will give full resistance from future rotavirus diseases, therefore, children may create the rotavirus illness more than once. Children who are not inoculated will, in general, have the most extreme side effects during their first rotavirus incident (2). Children who are inoculated are less inclined to get rotavirus and are bound to have milder side effects whenever tainted. The rotavirus vaccination program has reduced the prevalence and altered the seasonal patterns of rotavirus disease (2).

Low rotavirus vaccination coverage rates among children contribute to rotavirus outbreaks. Since rotavirus immunization inclusion lingers behind that of other youth antibodies, numerous kids stay helpless to serious rotavirus sickness. Rotavirus immunization is best against serious infection, therefore, even inoculated people may create gentle cases. Inoculation decreases transmission and might give circuitous insurance to unvaccinated people. Rotavirus flare-ups will keep on happening, in any case, even among profoundly inoculated populaces and among grown-ups (2).

Human services suppliers should keep on considering rotavirus disease in kids who present with intense gastroenteritis, while making solid suggestions to guardians for rotavirus inoculation and managing the antibodies to qualified newborn children as per CDC proposals (2). 
In Iran, the Ministry of Health and Medical Education has approved the rotavirus vaccine as the national Iranian EPI schedule in upcoming years. Thus, our medical personnel, especially pediatrician, advocate rotavirus vaccine as a standard preventive strategy against viral contamination in Iran.

\section{Footnotes}

Conflict of Interests: There is no conflict of interest.
Funding/Support: None declared.

\section{References}

1. Worls Health Organization. Rotavirus gastroenteritis. 2019. Available from: http://www.emro.who.int/health-topics/rotavirusgastroenteritis/disease-and-epidemiology.html.

2. Benjamin DH. Rotavirus Vaccination: 5 Things to Know. 2019. Available from: https://www.medscape.com/viewarticle/921833. 